\title{
2MASS NIR photometry for 693 candidate globular clusters in M 31 and the Revised Bologna Catalogue ${ }^{\star}$
}

\author{
S. Galleti ${ }^{1,2}$, L. Federici ${ }^{1}$, M. Bellazzini ${ }^{1}$, F. Fusi Pecci ${ }^{1}$, and S. Macrina ${ }^{2}$ \\ 1 INAF - Osservatorio Astronomico di Bologna, via Ranzani 1, 40127 Bologna, Italy \\ 2 Dipartimento di Astronomia, Università di Bologna, via Ranzani 1, 40127 Bologna, Italy \\ e-mail: [luciana;bellazzini;flavio]@bo.astro.it
}

Received 5 November 2003 / Accepted 2 December 2003

\begin{abstract}
We have identified in the 2MASS database 693 known and candidate globular clusters in M 31. The 2MASS $J, H, K$ magnitudes of these objects have been transformed to the same homogeneous photometric system of existing near infrared photometry of M 31 globulars, finally yielding $J, H, K$ integrated photometry for 279 confirmed $\mathrm{M} 31$ clusters, 406 unconfirmed candidates and 8 objects with controversial classification. Of these objects 529 lacked any previous estimate of their near infrared magnitudes. The newly assembled near infrared dataset has been implemented into a revised version of the Bologna Catalogue of M 31 globulars, with updated optical ( $U B V R I)$ photometry taken, when possible, from the most recent sources of CCD photometry available in the literature and transformed to a common photometric system. The final Revised Bologna Catalogue (available in electronic form) is the most comprehensive list presently available of confirmed and candidate M 31 globular clusters, with a total of 1164 entries. In particular, it includes 337 confirmed GCs, 688 GC candidates, 10 objects with controversial classification, 70 confirmed galaxies, 55 confirmed stars, and $4 \mathrm{H}$ II regions lying within $\sim 3^{\circ}$ from the center of the M 31 galaxy. Using the newly assembled database we show that the $V-K$ color provides a powerful tool to discriminate between M 31 clusters and background galaxies, and we identify a sample of 83 globular cluster candidates, which is not likely to be contaminated by misclassified galaxies.
\end{abstract}

Key words. galaxies: individual: M 31 - galaxies: star clusters - infrared: general - catalogs

\section{Introduction}

Globular clusters (GCs) are among the oldest astrophysical objects known and they are found in galaxies of all morphological types. Thus, globular clusters systems are extensively studied to recover fundamental information on the formation and early evolution of their host galaxies (Harris 1998a; Ashman \& Zepf 1998).

With present-day technology globular clusters can be fully resolved into stars down to below the Main Sequence (MS) Turn Off (TO) only if they reside in the neighbourhood of our own Galaxy (i.e., within $\sim 150 \mathrm{kpc}$, see Buonanno et al. 1998). The evolved populations (Red Giant Branch (RGB) and Horizontal Branch (HB)) may be resolved in the outer regions of globulars out to $\sim 1 \mathrm{Mpc}$ with the Hubble Space Telescope (see Rich et al. 2002, and references therein), and a handful of bright RGB stars have been resolved by Harris et al. (1998b) in a few clusters of the galaxy NGC 5128, at $\sim 4$ Mpc (but see Brown et al. 2003, for the improvements achievable with the

Send offprint requests to: $\mathrm{S}$. Galleti, e-mail: galleti@bo. astro.it

* Tables 2-4 are only available in electronic form at the CDS via anonymous ftp to cdsarc.u-strasbg.fr $(130.79 .128 .5)$ or via http://cdsweb.u-strasbg.fr/cgi-bin/qcat?J/A+A/416/917 new HST-ACS instrument). Therefore, to investigate the globular cluster system of even relatively nearby galaxies we have to rely on their integrated properties, e.g., magnitudes, colors and spectral indices (Harris 1998a).

In this framework, the study of the GC system of the Andromeda galaxy (M 31) is of special importance, since it is the only case in which the integrated properties of globular clusters, observed in conditions similar to those typical of farther galaxies, can be sensibly compared with their actual resolved stellar populations. Hence, apart from the obvious interest in the early history of the largest galaxy of the Local Group, the M 31 system is the ideal testbed to check our ability to obtain correct estimates of metallicity and ages of extragalactic globulars from their integrated colors and spectra.

The study of the M 31 GC system has a long and venerable history, starting with the first catalogues of candidates published by Hubble (1932) and Seyfert \& Nassau (1945). Since then, a number of authors have contributed to the detection, classification and analysis of M 31 globulars (see, e.g. Vetesnik 1962; Sargent et al. 1977; Crampton et al. 1985; Battistini et al. 1980, 1987, 1993; Mochejska et al. 1998; Barmby et al. 2000, 2001, 2002a; Barmby \& Huchra 2001, and references therein). In addition to the lists of candidates, these studies provided 
a large amount of data, mainly optical colors and spectral indices (i.e. 435 entries in the catalogue by Barmby et al. 2000). Near Infrared (NIR) $J, H, K$ photometry is available only for $\sim 260$ candidate clusters in the $J$ and $K$ passbands, and just for 127 in $H$ from the observations by Frogel et al. (1980); Sitko (1984); Bonoli et al. (1987, 1992); Cohen \& Matthews (1994); Barmby et al. (2000, 2001).

The Two Micron All Sky Survey (2MASS, Cutri et al. 2003), providing $J, H, K$ photometry of $99.998 \%$ of the sky down to $K \sim 15.5$ (completeness $>99 \%$ for $K \leq 14.3$ ), offers for the first time the opportunity of obtaining homogeneous NIR photometry for all the candidate GCs of M 31 within the limiting magnitude of the survey.

In the present paper we exploit this opportunity, providing $J, H$, and $K$ integrated magnitudes for 279 confirmed clusters $^{1}, 406$ unconfirmed candidates, and 8 objects for which the classification is controversial ${ }^{2}$. Near-IR photometry is less sensitive to interstellar extinction with respect to the classical optical colors and provides useful complementary information that may help for instance to disentangle the age-metallicity degeneracy (see Kissler-Patig et al. 2002; Puzia et al. 2002, and references therein).

The plan of the paper is the following: in Sect. 2 we present the master list of candidate M 31 GCs we have used as a reference catalogue (Master Catalogue), and we describe how the database of existing optical and NIR photometry has been assembled from the various sources in the literature; in Sect. 3 the actual search for counterparts of the Master Catalogue objects in the 2MASS database is described and discussed; Sect. 4 illustrates the power of the optical-NIR color index $V-K$ in the discrimination between bona fide globular clusters and background galaxies, in the range $V-K<3.0$; in Sect. 5 the Revised Bologna Catalogue of candidate globular clusters in M 31 is presented and described. The main results of the present analysis are summarized in Sect. 6.

\section{The master catalogue}

A reliable and complete catalogue of M 31 globulars based on modern CCD photometry has yet to be achieved even if limited to a quite bright threshold $V=18$, i.e., $M_{V} \sim-7$ at the M 31 distance. This situation arises because of: (a) the large field of view covered by the M 31 GC system (more than $9 \times 9 \mathrm{deg}$ in the sky, if extended out to $\sim 100 \mathrm{kpc}$ from the M 31 center), (b) the possible contamination by foreground stars, other objects within M 31 itself, and background galaxies (see Battistini et al. 1987, 1993, and references therein), and (c) the variable apparent size of the candidates (ranging from 2 up to $10 \mathrm{arcsec}$ ) often with a strong and highly variable photometric fore/background due to M 31 itself. Therefore, the available catalogues which we have assembled from different sources

\footnotetext{
${ }^{1}$ Confirmation of the classification is usually obtained by spectroscopic measures or by resolving the object into stars (see, e.g., Federici et al. 1990, 1993; Huchra et al. 1991; Barmby et al. 2000; Racine 1991; Racine \& Harris 1992).

2 Controversial objects are those that have been classified as "confirmed globulars" by some authors and as "confirmed galaxies" or stars by others.
}

are necessarily incomplete and, to some extent, contaminated. Moreover, both the photometric and the spectroscopic data are generally not homogeneous.

As a first step, we compiled a master list of the known candidates that has been reported in the literature up to 2003, June. The list of the original Bologna catalogue (Battistini et al. 1987) has been implemented with (i) the lists of cluster candidates detected near the nucleus by Battistini et al. (1993) and Aurière et al. (1992), (ii) the candidates found by Mochejska et al. (1998) and, finally, (iii) the list of new candidates found by Barmby \& Huchra (2001) searching M 31 HST/WFPC2 images in the HST archive.

The final database (hereafter the Master Catalogue, MC) includes 1164 entries: 337 confirmed GCs, 688 candidate GCs, 10 objects with controversial classification, 70 confirmed background galaxies, 55 confirmed stars, and 4 confirmed H II regions. These objects are spread out over an area of more than $3^{\circ} \times 3^{\circ}$ around the center of $\mathrm{M} 31$. The positions of all the entries are taken from the respective sources. We decided to include also previous candidates whose stellar or galactic nature has been (subsequently) observationally ascertained. Information about these objects may be useful (a) to avoid their re-discovery as candidate globulars and (b) to characterize the typical population of sources that may be misclassified as M 31 globulars.

\subsection{Optical photometry}

The available photometric measures have been secured by the various authors using different devices and techniques: CCD and photoelectric photometry, photographic plates and, for several candidates, visual photometry obtained by eye. To assemble all this material as coherently as possible we ranked the sources of M 31 GCs photometry according to the adopted technique, choosing CCD photometry whenever available. Lacking CCD photometry, photoelectric photometry was preferred to photographic photometry and the latter was preferred to eye estimates based on photographic plates. We chose to include eye estimates to provide, at least, a guide for future observations of the candidates which lack any instrumental photometry. When one cluster was present in two different sets of CCD photometry the source with the lower photometric uncertainty and/or providing the larger number of entries was preferred.

In order to obtain a Master Catalogue with photometric measures as homogeneous as possible we have taken as a photometric reference the dataset by Barmby et al. (2000, their Table 3), that gives homogeneous UBVRI data for 260 confirmed GC and candidates. All other catalogues considered in the present compilation have been transformed to this reference list by applying the offsets we derived from the objects in common between the considered catalogue and the list of Barmby et al. (see Macrina 2004, for details). The derived offsets are reported in Table 1 and are in good agreement with what found by Barmby et al. (2000, see their Table 4). In case of duplicated CCD observations from Barmby et al. (2000) and Barmby \& Huchra (2001) the average value has been adopted. The same approach has been adopted for duplicate photoelectric 
Table 1. Photometric offsets.

\begin{tabular}{|c|c|c|c|c|c|c|}
\hline Data & Catalog & $\overline{\Delta U}$ & $\Delta B$ & $\overline{\Delta V}$ & $\overline{\Delta R}$ & $\overline{\Delta I}$ \\
\hline \multicolumn{7}{|c|}{ CCD: } \\
\hline & B93 & $\ldots$ & -0.37 & 0.00 & -0.42 & 0.00 \\
\hline & RHH92/94 & $\ldots$. & 0.00 & 0.00 & 0.00 & .... \\
\hline & M98 & $\ldots$. & $\ldots$. & 0.00 & $\ldots$. & 0.00 \\
\hline \multicolumn{7}{|l|}{ PE: } \\
\hline & SL83 & 0.00 & 0.00 & 0.00 & $\ldots$. & $\ldots$ \\
\hline & SL85+ & 0.00 & 0.00 & 0.00 & $\ldots$. & $\ldots$ \\
\hline & B87 & $\begin{array}{c}0.00(\text { if } U \leq 17.9) \\
-0.27 U+4.8(\text { if } U>17.9)\end{array}$ & $\begin{aligned} 0.00 & \text { (if } B \leq 18) \\
-0.31 B & +5.64(\text { if } B>18)\end{aligned}$ & $\begin{aligned} 0.00 & (\text { if } V \leq 16.5) \\
-0.13 V+2.17 & (\text { if } V>16.5)\end{aligned}$ & $-0.03 r_{\mathrm{c}}+0.16$ & $*$ \\
\hline
\end{tabular}

Reference: RHH92 Reed et al. (1992); RHH94 Reed et al. (1994); B93 Battistini et al. (1993); M98 Mochejska et al. (1998); SL83 Sharov \& Lyutyi (1983); SL85+ Sharov \& Lyutyi (1985) and succeeding papers; B87 Battistini et al. (1987); (*) unpublished I photographic magnitudes (Federici \& Fusi Pecci, private communication). The indicated offset have been applied to the photometries of the different sources to transform them into the photometric system of Barmby et al. (2000).

measures from Sharov (1973) and Sharov \& Lyutyi (1983). No transformation is feasible for the $V$ eye magnitudes of the 132 D class objects from Battistini et al. (1980) since this data set has too few objects (15) in common with other catalogues to derive a reliable transformation. 26 objects taken from the list of Crampton et al. (1985) have no photometry from other sources, hence they lack optical photometry in the JohnsonCousins system and the magnitudes provided by Crampton cannot be consistently transformed to the photometric reference adopted here.

Concerning the photometric errors, one has to refer to the original sources. Typical internal errors for the CCD and photoelectric magnitudes are less than 0.05 mag (0.08 in $U$ ); however, the photometry of candidates affected by a strong background in the inner M 31 regions typically has larger errors. For the photographic magnitudes the typical errors range from 0.05 up to $0.20 \mathrm{mag}$, as shown by the comparisons with the CCD data in common. For the 15 objects with both eye-estimates and CCD or photoelectric photometry, comparison of $V$ magnitudes suggests that the typical uncertainty of the eye-estimates is \pm 0.5 mag (Macrina 2004).

\subsection{Near IR photometry}

Though the observations were carried out with different setups (telescopes, filters, etc.), the existing NIR photometric data sets (Frogel et al. 1980; Sitko 1984; Bonoli et al. 1987, 1992; Cohen \& Matthews 1994) are in agreement to within the errors (see Barmby et al. 2000, 2001). These errors are fairly large: $\sim 0.1 \mathrm{mag}$ in $J$, and $\sim 0.2 \mathrm{mag}$ in $H$ and $K$. Hence the $J, H, K$ magnitudes available before 2MASS can be considered as a virtually homogeneous set. In the compilation of the Master Catalogue, as a first step, we have therefore adopted for each object the average values of all the available measurements, obtaining $J$ and $K$ magnitudes for $\sim 260$ objects and $H$ magnitudes for 127 objects.

\subsection{Astrometry and identifications}

The coordinates of the objects included in the MC are taken from the original sources. They were measured with slightly different reference frames and are thus affected by different internal and systematic uncertainties. For this reason, performing the cross-correlation of the MC with the 2MASS database (described in Sect. 3), we had to check several cases of objects whose identification was not straightforward (see below). This analysis led to a few corrections of the original MC that are described in the present section.

Several direct checks carried out during the preparation of HST and spectroscopic observations (see, i.e. Fusi Pecci et al. 1996; Rich et al. 2003; Barmby et al. 2002a) have confirmed that the average astrometric accuracy of the coordinates in the Battistini et al. $(1987,1993)$ catalogue is better than $1^{\prime \prime}$.

For all A, B and confirmed C class objects from Battistini et al. (1987) with no 2MASS counterpart within 1" (see below) the coordinates have been checked on the Digitized Sky Survey (DSS-2) or on HST archive WFPC2/STIS frames, when available. This last check was needed for the objects near the bulge or in regions with a very high background, which cannot be seen on the DSS-2 images. The MC coordinates have been verified also for all the objects that had $J H K$ photometry in the MC, but that were not found in the 2MASS database (see below). The identification of these objects has been performed directly on the available images and their listed positions has been accordingly revised.

In a few cases, we have found a discrepancy between the coordinates reported in the Battistini et al. (1987) catalogue and the corresponding finding charts. In particular, B472 is the brightest object $\sim 18^{\prime \prime}$ SE of what reported in the Battistini et al. (1987) finding chart, B453 is 27" NE, B450 is $\sim 1^{\prime} \mathrm{SE}$, and B284 is $\sim 3^{\prime}$ S. Moreover, the coordinates of B288 and B142 in Battistini et al. (1987) are wrong, the correct values are reported in Table 2.

In Battistini et al. (1987) there are also a few wrong crossidentifications with previous catalogues. In Table VII, G162 corresponds to SH11 and G171 to SH12; as noted by Sharov \& Lyutyi (1983) G162 and G171 actually are SH12 and SH11, 
respectively. Moreover, in Table IV of Racine (1991) the object 231D-DAO61 is actually 230D-DAO61 and in Table V(b) DAO95 is DAO96.

Finally, the visual check carried out on the HST WFPC2/STIS archival frames allowed a better classification of a few candidates. B123 and B196 are clearly resolved into stars by HST, hence they have been classified as confirmed globular clusters; B261 is recognized as a foreground star while B329 is a background galaxy. B445 and NB76 appear as good GC candidates, while B430 is probably a star, but the classification is not completely unambiguous in these cases.

\section{M 31 cluster candidates in the 2MASS database}

\subsection{MASS IR photometry of the objects}

The classification of unresolved globular clusters is a very hard task. Because of the round and compact appearance of unresolved GCs at the typical resolution of ground-based images $\left(\sim 1^{\prime \prime}\right)$, both distant galaxies and foreground stars can be misclassified as GCs. Given the low spatial resolution of the survey, the automated classification pipeline of 2MASS has assigned the M 31 GCs either to the Point Source Catalogue (PSC) or to the Extended Source Catalog (XSC), depending on their apparent sizes. Therefore we decided to search the objects of the MC in both catalogues.

We selected from the 2MASS All-Sky Data Release all the sources within an area of $4^{\circ} \times 4^{\circ}$ centered on M 31 from both the PSC and the XSC data-sets, excluding all entries flagged as low-quality or contaminated by nearby sources. A general astrometric transformation between the MC and the 2MASS catalogues was found using the many entries in common as reference. The astrometric solutions were refined in repeated iterations until a typical residual $<1^{\prime \prime}$ was achieved. The final cross-correlation between the MC and the PSC provided 597 identifications, while 179 MC objects have been crossidentified in the XSC. In both cases the identification was considered safe if a 2MASS source was found within 1 " from the position of a MC entry (see Fig. 1). The identification of the sources found at distances larger the $1^{\prime \prime}$ from one MC entry has been checked by visual inspection and has been accepted only if there was no possible ambiguity. Note that most of the identifications with residuals larger than $1^{\prime \prime}$ regards XSC entries. Of the total of 776 identified objects, 693 are confirmed or candidate globular clusters, the remaining 83 are confirmed stars, $\mathrm{H}$ II regions or galaxies ${ }^{3}$. More than one hundred of the confirmed or candidate GCs identified in the 2MASS databases have previous NIR photometry. In particular, 247 objects have

\footnotetext{
${ }^{3}$ The reported number of $\mathrm{MC}$ objects actually identified in the 2MASS databases depends on the considered passband. For example the total number of confirmed and candidate clusters who have a couterpart in 2MASS are 693 in $J, 675$ in $H$ and 620 in $K$; the number of objects who lacked any previous IR photometry are 529, 637 and 463 if we consider the $J, H$ and $K$ passband, respectively. For brevity and to avoid confusion, all over the paper we report only the numbers referring to the $J$ band measures. The corresponding numbers in the other passbands are similar, the exact numbers can be obtained by inspecting the catalogues presented in Tables 2, 3 and 4.
}

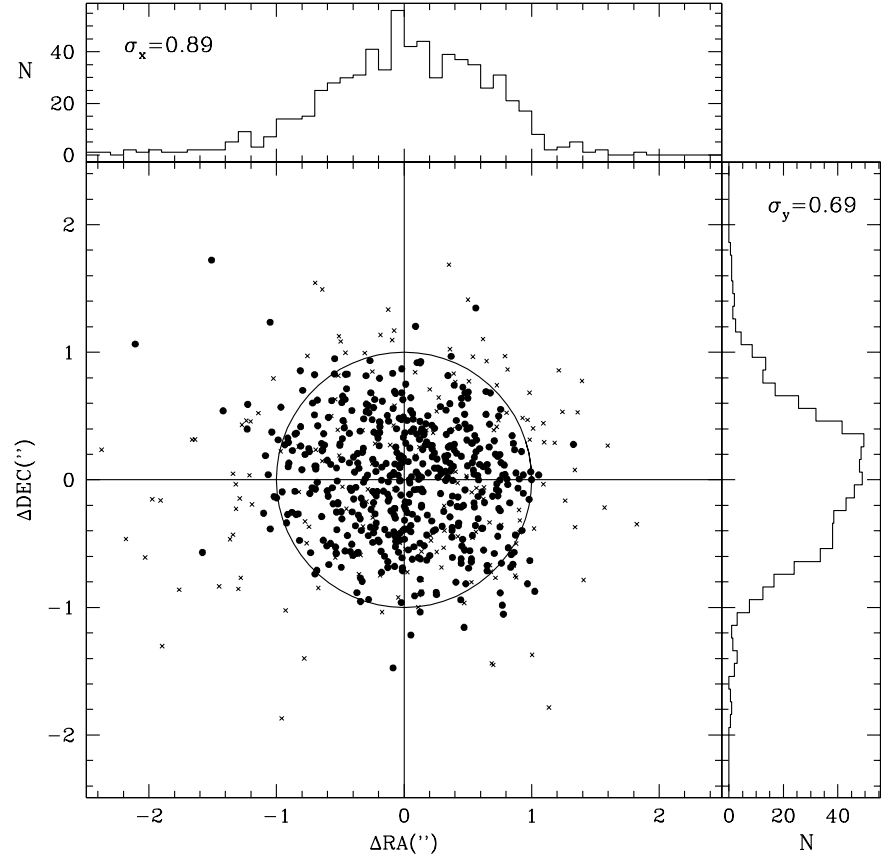

Fig. 1. Residuals of the astrometric solution for PSC (filled circles) and XSC (crosses) sources. The histograms show the RMS of RA (top panel) and Dec (right panel). A circle of radius $r=1^{\prime \prime}$ has been superposed to the plot as a reference. The standard deviation of the distributions of the residuals in each directions (histograms) are also reported within the corresponding boxes.

previous $J$ photometry, 122 have previous $H$ photometry and 236 have previous $K$ photometry. This large set of objects in common allows a sensible comparison between the different photometries (see below). There are $\sim 400 \mathrm{MC}$ entries for which we cannot find a counterpart in the 2MASS catalogues. These include $\sim 20$ (the exact numbers depends on the considered passband) objects with pre-2MASS NIR photometry. Almost all of these are fainter than the limiting magnitude of the 2MASS survey. Finally, the MC contains 88 entries (from different sources, e.g. Battistini et al. 1987, 1993; Crampton et al. 1985; Aurière et al. 1992) that have no optical photometry. These include 7 confirmed GC, 56 GC candidates and 25 confirmed stars/galaxies/H II regions. We provide here $J, H$, $K$ photometry from 2MASS for 30 of them, 19 of which being confirmed or candidate clusters.

\subsection{The IR photometric system and the adopted JHK magnitudes}

After a set of extensive tests of the different kind of magnitudes provided in the PSC and XSC catalogues (e.g. isophotal, Point Spread Function fitting, aperture), we decided to rely on the aperture photometry in both cases. Unfortunately, magnitudes measured with the same aperture were not available for both catalogues; hence our final choice was to use the aperture photometry within $r=4^{\prime \prime}$ for the PSC and $r=5^{\prime \prime}$ for the XSC. All the 2MASS magnitudes have then been transformed to the CIT photometric system (Frogel et al. 1978; Elias 1982, 1983) using the color transformations provided by Carpenter (2001). 



Fig. 2. Comparison between the 2MASS photometry and previous NIR-photometry from the Master Catalogue. Left panels: 2MASS sources identifyied in the XSC. Right panels: 2MASS sources identifyied in the PSC. The extended source are in left panel and the points source in righ panel.

Figure 2 shows the comparison between the 2MASS photometry and the previous NIR photometries as adopted in the MC for the sources identified in the XSC (left panels) and in the PSC (right panels). In all cases the average absolute difference is $<0.1 \mathrm{mag}$ and the rms scatter around the mean is $<0.2 \mathrm{mag}$. No residual color effect has been detected.

There are small systematic shifts determined from Fig. 2, probably due to the difference in the adopted radius of the aperture. These have thus been applied to the 2MASS photometry to put all of the measures to the same photometric calibration adopted in the MC.

In Fig. 3 the $J H K$ photometry of the total sample (obtained by merging the corrected magnitudes from the PSC and XSC) are compared with the previous NIR photometry from the MC. As can be seen, (a) the corrected magnitudes from both the PSC and the XSC form a homogeneous set, and (b) for the vast majority of the candidates the difference between corrected 2MASS photometry and previous photometries is less then $\pm 0.2 \mathrm{mag}$ in all passbands, i.e., of the order of the intrinsical uncertainties of pre-2MASS NIR measures. The few objects displaying a difference larger that $\simeq 0.5 \mathrm{mag}$ has been checked by visual inspection of DSS images. All of them lie within $\sim 8-11^{\prime \prime}$ of another source of similar or larger luminosity, hence their magnitude estimates may be influenced by the contamination of the close companions. The above procedure has provided $J H K$ photometry for 529 objects for which no previous NIR photometry was available. In particular, the final catalogue contains homogeneous NIR data for 291 confirmed GCs, 412 candidates, 8 controversial objects, 58 confirmed galaxies, 23 confirmed stars and $3 \mathrm{H}$ II regions.

Fig. 3. Comparison between the photometry of the final sample (corrected PSC+XSC) and previous NIR-photometry. The dashed lines encloses the range of $\pm 0.2 \mathrm{mag}$.

In conclusion, the NIR photometric dataset of the M 31 GC candidates has been nearly tripled by the present work.

\section{NIR-optical color-color diagrams: A powerful tool for a preliminar classification}

The knowledge of integrated colors offers a wealth of information on any stellar population, and in particular, may be quite useful for classification purposes. Here we show that the available data on integrated colors may be used in many cases to discriminate between star clusters and background galaxies thanks to the large baseline provided by optical-infrared colors.

Various color-color diagrams with $V-K$ vs. optical colors are presented in Fig. 4 where small points are confirmed M 31 globulars, and filled circles are the confirmed galaxies that are present in our Master Catalogue. These galaxies should be typical of the background galaxies that can contaminate the set of candidate M 31 GCs. In each panel of Fig. 4 (as well as in Fig. 5 below) the arrow indicates the reddening vector corresponding to $E(B-V)=0.1$ to illustrate quantitatively the effects of the interstellar exctinction.

There are two remarkable features in Fig. 4: (1) the confirmed GCs (points) span a range of more than 3 magnitudes in $V-K$, much larger than in optical colors (also due to the larger impact of the reddening in $V-K$ ); (2) the vast majority of confirmed galaxies (filled circles) has $V-K \geq 3.0$, while $\sim 50 \%$ of confirmed globulars are bluer than this limit, reaching colors as blue as $V-K \sim 1.2$.

Therefore the $V-K$ colors provide a useful discriminant to separate good candidate clusters from background galaxies. This should lead to higher efficiency in observational programs 


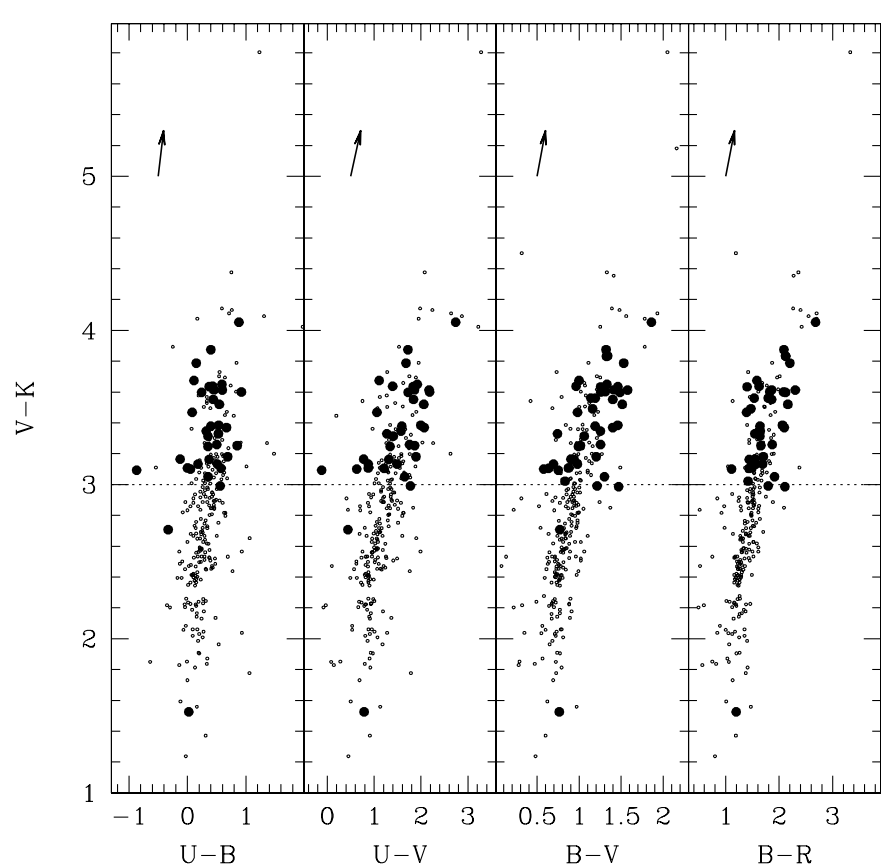

Fig. 4. $V-K$ vs. various optical colors for the confirmed globular clusters (small points) and for the confirmed galaxies (filled circles).

aimed at assessing the classification of clusters in $M$ 31, since it is very unlikely that a candidate having $V-K<3.0$ could be a misclassified background galaxy. On the other hand, foreground stars that may be erroneously classified as candidate M 31 globulars span the whole range of $V-K$ colors covered by confirmed clusters. Therefore, while the above criterion is very valuable for the galaxy/cluster discrimination, it is ineffective in the star/cluster separation.

It may also be useful to note that most of the clusters having $V-K \geq 4.0$ lie in a region of the M 31 disk that seems to be affected by higher extinction (Barmby et al. 2000). The isolated cluster at $V-K>5.0$ is B037. Its very red color is probably due to a color excess $E(B-V)>1.0 \mathrm{mag}$ (in which case this may be the brightest globular of the whole M 31 system, see Barmby et al. 2002b, for discussion and references), or to contamination by a bright and cold star accidentally superposed to the cluster. The confirmed galaxy at $V-K \sim 2.75$ is B136D (confirmed by Huchra et al. 1991), the one at $V-K \sim 1.5$ is B390 (classified as galaxies by Racine 1991).

Making use of the indications drawn from the plots presented in Fig. 4 for the confirmed clusters and galaxies, one can apply the above criterion to get useful hints on the nature of candidates whose classification is uncertain. In Fig. 5 we show the same color-color diagrams as in Fig. 4 for the notyet-confirmed candidate GCs (small open circles) in our Master Catalogue and for the four controversial objects (filled squares) classified as "cluster" by some authors and as "galaxy" by others. It can be readily seen that most candidates have colors consistent with those typical of background galaxies $(V-K \geq 3.0)$, hence it can be reasonably deduced that many of them are in fact misclassified galaxies. On the other hand, 83 candidates have $1.2 \leq V-K<3.0$ and, according to the above discussion, the contamination of this sub-sample by background galaxies



Fig. 5. $V-K$ vs. optical colors for the not-yet-confirmed candidates globular cluster (small open circles) and for controversial objects that have been classified as clusters by some authors and as galaxies by others (filled squares).

should be negligible. A spectroscopic campaign on these blue candidates would probably be very rewarding in terms of confirmation of bona fide clusters per observed candidate. On the other hand, it has to be recalled that the criterion introduces an obvious bias against intrinsically red and/or highly reddened clusters, hence it cannot be considered as a suitable method to select complete samples of candidate clusters.

The controversial candidates G268, B409, G355 and B140 have been classified as galaxies by Racine (1991), based on high resolution ground based imaging, while Federici et al. (1990); Huchra et al. (1991); Perrett et al. (2002) classified them as globular clusters on the basis of spectroscopical observations. All these candidates have $V-K<3.0$, hence their $V-K$ colors seem to confirm the classification by Federici et al. (1990) and Perrett et al. (2002). For the other four controversial candidates not mentioned here, the controversy is between the classification as a cluster or as a star, hence the doubt cannot be resolved with the presently adopted criterion.

\section{The Revised Bologna Catalogue}

The MC described in Sect. 2, implemented with the new set of NIR magnitudes obtained from 2MASS constitutes a new version of the Battistini et al. (1987) catalogue: the Revised Bologna Catalogue of candidate GCs in M 31. The Revised Bologna Catalogue includes 337 confirmed GCs, 688 GC candidates, 10 objects with controversial classification, 70 confirmed background galaxies, 55 confirmed stars and $4 \mathrm{H}$ II regions. The whole catalogue is organized in three different tables, all of them made available at the CDS in electronic form. 
Identifications and adopted coordinates are reported in Table 2, that contains the following columns:

- Column 1: designation derived from the object coordinates, following Barmby \& Huchra (2001) (M 31JHHMMSS+DDMMSS);

- Column 2: designation: B and BxxxD from Battistini et al. (1980, 1987), G from Sargent et al. (1977), V from Vetesnik (1962), DAO from Crampton et al. (1985), NB from Battistini et al. (1993), AU from Aurière et al. (1992), M from Mochejska et al. (1998), BH from Barmby \& Huchra (2001), SH from Sharov \& Lyutyi (1983), BA from Baade \& Arp (1964);

- Columns 3-12: different identifications, respectively from Battistini et al. (1987, 1993); Sargent et al. (1977); Vetesnik (1962); Aurière et al. (1992); Crampton et al. (1985); Baade \& Arp (1964); Sharov (1973); Mochejska et al. (1998); Barmby \& Huchra (2001);

- Column 13: classification flag describing the nature of the entry (1: confirmed GC, 2: GC candidate, 3 : controversial object, 4: confirmed galaxy, 5: confirmed H II region, 6: confirmed star);

- Column 14: classification from the original papers (Battistini et al. 1987; Mochejska et al. 1998; Barmby \& Huchra 2001);

- Column 15: spectroscopic confirmation: C - cluster, G galaxy, S - star, H - H II region;

- Column 16: confirmation via high resolution imaging: C cluster, G - galaxy, S - star, H - H II region;

- Column 17: comments and references;

- Columns 18 and 19: RA, Dec (J2000) from the original catalogues or from DSS-2 or from HST/WFPC2 images (see Sect. 3);

- Column 20: sources of the adopted coordinates.

The adopted photometric data have been collected and are listed in Table 3 for all the confirmed and candidate globular clusters, and in Table 4 for all the objects confirmed to be galaxies, stars or $\mathrm{H}$ II regions. The two tables have the following columns:

- Column 1: designation, as in Table 2;

- Column 2: classification, as Col. 12 in Table 2;

- Columns 3-10: $U, B, V, R, I, J, H, K$ magnitudes (see Sect. 2);

- Column 14: sources of the adopted optical data and NIR pre-2MASS.

The entries associated to the object listed in Table 4 are reported at the end of Table 2. In this way Table 2 can be easily combined with Tables 3 and 4 to obtain tables including positions and photometric properties of "candidate GC only" and "confirmed contaminant objects only".

\section{Summary and future prospects}

We have compiled a Master Catalogue of M 31 globular cluster candidates including 1164 entries and we have searched the counterparts of the MC objects in the NIR 2MASS databases.
We have found 776 2MASS counterparts, 693 of which correspond to confirmed or candidate M 31 GCs. In this way we have provided integrated $J, H, K$ magnitude for 529 objects for which no previous NIR photometry was available.

Moreover, we have collected and revised all the available photometries, yielding a final adopted list of UBVRIJHK magnitudes as homogeneous as possible. The classification of a few candidates has been assessed by inspection of HST images and a few controversial cases have been reconsidered. With all the newly collected material, we have updated the original Bologna Catalogue (Battistini et al. 1987) so obtaining the Revised Bologna Catalogue (RBC). The RBC is the most comprehensive list presently available of candidate M 31 clusters, including 1035 confirmed and candidate GCs, 70 confirmed galaxies, 55 confirmed stars, and $4 \mathrm{H}$ II regions previously considered possible GC candidates, for a total of 1164 entries. The Bologna Revised Catalogue is currently in use to prepare observational programs aimed at increasing the sample of bona-fide M 31 globular clusters and to study the integrated properties of the whole globular clusters system of M 31 .

It has also been shown that optical-infrared colors may be very valuable in the discrimination between background galaxies and bona fide globular clusters for $V-K<3.0$. According to this criterion, we can extract from the RBC a sub-sample of 83 objects whose contamination by misclassified background galaxies is (probably) very low.

Acknowledgements. We are very grateful to R.T. Rood for a critical reading of the original manuscript and to an anonymous Referee for her/his useful comments and suggestions. This publication makes use of data products from the Two Micron All Sky Survey, which is a joint project of the University of Massachusetts and the Infrared Processing and Analysis Center/California Institute of Technology, funded by the National Aeronautics and Space Administration and the National Science Foundation. Part of this work is based on observations made with the NASA/ESA Hubble Space Telescope, obtained from the data archive at the Space Telescope Science Institute. STScI is operated by the Association of Universities for Research in Astronomy, Inc., under NASA Contract NAS 5-26555. This work was supported by a fellowship (S.G.) from the Consorzio Nazionale Astronomia ed AstrofisicaCNAA and contributions from MIUR-COFIN and Agenzia Spaziale Italiana-ASI.

\section{References}

Ashman, K. M., \& Zepf, S. 1998, Globular Cluster Systems (New York: Cambridge Univ. Press)

Aurière, M., Coupinot, G., \& Hecquet, J. 1992, A\&A, 256, 95

Baade, W., \& Arp, H. 1964, ApJ, 139, 1027

Battistini, P. L., Bonoli, F., Braccesi, A., et al. 1980, A\&AS, 42, 357

Battistini, P. L., Bonoli, F., Braccesi, A., et al. 1987, A\&AS, 67, 447

Battistini, P. L., Bonoli, F., Casavecchia, M., et al. 1993, A\&A, 272, 77

Barmby, P., Huchra, J. P., Brodie, J. P., et al. 2000, AJ, 119, 727

Barmby, P., Huchra, J. P., \& Brodie, J. P. 2001, AJ, 121, 1482

Barmby, P., \& Huchra, J. P. 2001, AJ, 122, 2458

Barmby, P., Holland, S., \& Huchra, J. P. 2002, AJ, 123, 1937

Barmby, P., Perrett, K. M., \& Bridges, T. J. 2002, MNRAS, 329, 461

Bonoli, F., Delpino, F., Federici, L., \& Fusi Pecci, F. 1987, A\&A, 185, 25 
Bonoli, F., Delpino, F., Federici, L., Fusi Pecci, F., \& Longmore, A. J. 1992, A\&AS, 96, 163

Brown, G. M., Ferguson, H. C., Smith, E., et al. 2003, ApJ, 592, L17

Buonanno, R., Corsi, C. E., Zinn, R., et al. 1998, ApJ, 501, L33

Carpenter, J. M. 2001, AJ, 121, 2851

Cohen, J. G., \& Matthews, K. 1994, AJ, 108, 128

Crampton, D., Cowley, A. P., Schade, D., \& Chayer, P. 1985, ApJ, 288,494

Cutri, et al. 2003, Explanatory Supplement to the 2MASS All Sky Data Release, http://www.ipac.caltech.edu/2mass/ releases/allsky/doc/explsup.html

Elias, J. H., Frogel, J. A., Matthews, K., \& Neugebauer, G. 1982, AJ, 87, 1029 and 1893

Elias, J. H., Frogel, J. A., Hyland, A. R., \& Jones, T. J. 1983, AJ, 88, 1027

Federici, L., Fusi Pecci, F., \& Marano, B. 1990, A\&A, 236, 99

Federici, L., Bonoli, F., Ciotti, L., et al. 1993, A\&A, 274, 87

Frogel, J. A., Persson, S. E., \& Cohen, J. G. 1980, ApJ, 240, 785

Frogel, J.A., Persson, S.E., Matthews, K., \& Aaronson, M. 1978, ApJ, 220, 75

Fusi Pecci, F., Buonanno, R., Cacciari, C., et al. 1996, AJ, 112, 1461

Harris, W. E. 1998a, in Star Clusters, Saas-Fee Advanced Course 28, ed. L. Labhardt, \& B. Binggeli (Springer), 223

Harris, G. L. H., Poole, G. B., \& Harris, W. E. 1998b, AJ, 116, 2866
Hubble, E. P. 1932, ApJ, 76, 44

Huchra, J. P., Brodie, J. P., \& Kent, S. 1991, ApJ, 370, 495

Kissler-Patig, M., Brodie, J. P., \& Minniti, D. 2002, A\&A, 391, 441

Macrina, S. 2004, Tesi di Laurea, Bologna University

Mochejska, B. J., Kaluzny, J., Krockenberger, M., Sasselov, D. D., \& Stanek, K. Z. 1998, Acta Astron., 48, 455

Perrett, K. M., Bridges, T. J., Hanes, D. A., et al. 2002, AJ, 123, 2490

Puzia, T. H., Zepf, S. E., Kissler-Patig, M., et al. 2002, A\&A, 391, 453

Racine, R. 1991, AJ, 101, 865

Racine, R., \& Harris, W. E. 1992, AJ, 104, 1068

Reed, B. C., Harris, G. L. H., \& Harris, W. E. 1992, AJ, 103, 824

Reed, B. C., Harris, G. L. H., \& Harris, W. E. 1994, AJ, 107, 555

Rich, R. M., et al. 2003, in preparation

Rich, R. M., Corsi, C. E., Bellazzini, M., et al. 2002, in Extragalactic Star Clusters, ed. D. Geisler, E. K. Grebel, \& D. Minniti (San Francisco: ASP), IAU Symp., 207, 140

Sargent, W. L. W., Kowal, C. T., Hartwick, F. D. A., \& van den Bergh, S. 1977, AJ, 82, 947

Seyfert, C. K., \& Nassau, J. J. 1945, ApJ, 102, 377

Sharov, A. S. 1973, Sov. Astron. A.J.,17,174

Sharov, A. S., \& Lyutyi, V. M. 1983, Ap\&SS, 90, 371

Sharov, A. S., \& Lyutyj, V. M. 1985, AstL, 11, 248

Sitko, M. L. 1984, ApJ, 286, 209

Vetesnik, M. 1962, BAICz, 13, 180 